\title{
Open Fetal Microneurosurgery for Intrauterine Spina Bifida Repair
}

\author{
Rogelio Cruz-Martínez ${ }^{a, b, c}$ Felipe Chavelas-Ochoad

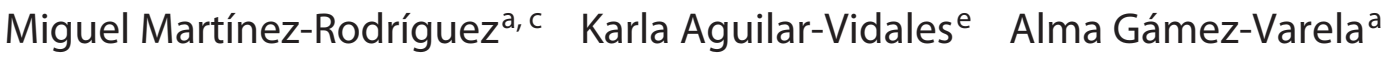 \\ Jonahtan Luna-García ${ }^{a}$ Hugo López-Briones ${ }^{a}$ Joel Chávez-Vega e \\ Ángel Augusto Pérez-Calatayud ${ }^{f}$ Manuel Alejandro Díaz-Carrillo ${ }^{f}$

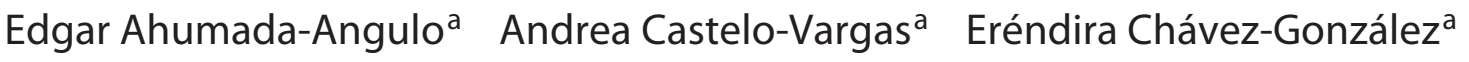 \\ Israel Juárez-Martínez ${ }^{\mathrm{a}}$ Rosa Villalobos-Gómez ${ }^{\mathrm{a}} \quad$ Carlos Rebolledo-Fernández $^{\mathrm{g}}$ \\ aPrenatal Diagnosis and Fetal Surgery Center, Fetal Medicine Mexico and the Fetal Medicine Foundation of Mexico, \\ Queretaro, Mexico; 'b Instituto de Ciencias de la Salud (ICSa), Universidad Autónoma del Estado de Hidalgo (UAEH), \\ Hidalgo, Mexico; 'Department of Fetal Surgery, Hospital de Especialidades del Niño y la Mujer Dr. Felipe Núñez \\ Lara, Queretaro, Mexico; ${ }^{\mathrm{d} D e p a r t m e n t}$ of Pediatric Neurosurgery, Hospital de Especialidades del Niño y la Mujer \\ Dr. Felipe Núñez Lara, Queretaro, Mexico; 'Department of Anesthesiology, Hospital de Especialidades del Niño y la \\ Mujer Dr. Felipe Núñez Lara, Queretaro, Mexico; ${ }^{f}$ Department of Maternal Intensive Care, Hospital de Especialidades \\ del Niño y la Mujer Dr. Felipe Núñez Lara, Queretaro, Mexico; '9Department of Maternal Fetal Medicine, Hospital de \\ Especialidades del Niño y la Mujer Dr. Felipe Núñez Lara, Queretaro, Mexico
}

\section{Keywords}

Fetal surgery · Microneurosurgery · Minihysterotomy · Myelomeningocele $\cdot$ Spina bifida

\begin{abstract}
Objectives: The aim of the study was to describe the feasibility of open fetal microneurosurgery for intrauterine spina bifida (SB) repair and to compare perinatal outcomes with cases managed using the classic open fetal surgery technique. Methods: In this study, we selected a cohort of consecutive fetuses with isolated open SB referred to our fetal surgery center in Queretaro, Mexico, during a 3.5-year period (20162020). SB repair was performed by either classic open surgery (6- to 8-cm hysterotomy with leakage of amniotic fluid, which was replaced before uterine closure) or open microneurosurgery, which is a novel technique characterized by a 15- to 20-mm hysterotomy diameter, reduced fetal manipulation by fixing the fetal back, and maintenance of normal
\end{abstract}

amniotic fluid and uterine volume during the whole surgery. Perinatal outcomes of cases operated with the classic open fetal surgery technique and open microneurosurgery were compared. Results: Intrauterine SB repair with a complete 3-layer correction was successfully performed in 60 cases either by classic open fetal surgery $(n=13)$ or open microneurosurgery $(n=47)$. No significant differences were observed in gestational age (GA) at fetal intervention (25.4 vs. 25.1 weeks, $p=0.38$ ) or surgical times (107 vs. $120 \mathrm{~min}, p=0.15$ ) between both groups. The group with open microneurosurgery showed a significantly lower rate of oligohydramnios ( 0 vs. $15.4 \%, p=0.01)$, preterm rupture of the membranes $(19.0$ vs. $53.8 \%, p=0.01$ ), higher GA at birth (35.1 vs. 32.7 weeks, $p=0.03$ ), lower rate of preterm delivery $<34$ weeks ( 21.4 vs. $61.5 \%, p=0.01$ ), and lower rate of perinatal death (4.8 vs. $23.1 \%, p=0.04)$ than the group with classic open surgery. During infant follow-up, the rate of hydrocephalus requiring ventriculoperitoneal shunting was similar between both groups (7.5 vs. $20 \%, p=0.24)$. All patients showed an intact

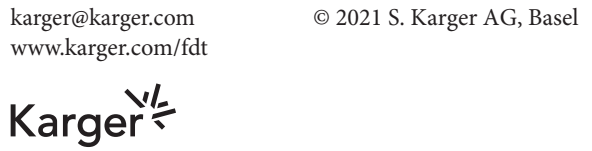


hysterotomy site at delivery. Conclusion: Intrauterine spina repair by open fetal microneurosurgery is feasible and was associated with better perinatal outcomes than classic open fetal surgery.

(C) 2021 S. Karger AG, Basel

\section{Introduction}

Spina bifida (SB) is a neural tube malformation that represents the most common severely disabling congenital defect. It complicates about 1 in 2,000 live births and is associated with major disabilities such as motor dysfunction or paralysis, mental retardation, hydrocephalus requiring ventriculoperitoneal shunting, bowel and bladder dysfunction, and long-term neurological handicaps [1]. Since fetal brain damage is progressive during gestation due to the chronic exposure of neural tissue to the amniotic fluid, fetal surgery for prenatal myelomeningocele (MMC) repair was then introduced in an attempt to preserve neurological functions [2]. In a randomized double-blind control trial, open fetal surgery demonstrated to be of benefit in improving hindbrain herniation, ventriculoperitoneal shunting, motor function, and cognitive outcomes in comparison to cases managed with postnatal surgery [3].

However, the aforementioned fetal intervention is also associated with potential maternal and fetal risks such as preterm delivery, placental abruption, preterm rupture of the membranes, perinatal mortality, uterine bleeding requiring blood transfusion, uterine dehiscence at delivery, and higher risk of preterm delivery even in subsequent pregnancies [4-6], and therefore, the design of novel minimally invasive techniques is in the research agenda of different fetal surgery centers [7].

Previous studies have designed minimally invasive approaches for fetal MMC repair through fetal endoscopy with or without assisted maternal laparotomy. However, 2 meta-analyses have suggested that in comparison to open fetal surgery, fetal endoscopy is associated with a higher risk of preterm delivery, preterm prelabor rupture of membranes (PPROM), and perinatal deaths mainly because of the higher surgical time that is required for MMC repair $[8,9]$. Initial experience has also suggested that decreasing the hysterotomy diameter during fetal intervention may improve perinatal outcomes without altering the neurological results $[10,11]$. Thus, in this study, we aimed to describe the feasibility of open microneurosurgery for intrauterine SB repair, a novel alternative technique characterized by a minimal uterine incision of
$15-20 \mathrm{~mm}$, reduced fetal manipulation, and maintenance of normal amniotic fluid volume during the whole intervention. We also compared the perinatal outcomes of such cases with those previously operated with the classic open fetal surgery technique in our center.

\section{Methods}

\section{Subjects}

Between December 2016 and May 2020, a prospective cohort of singleton fetuses with confirmed lumbosacral open SB were selected for fetal surgery at Hospital de Especialidades del Niño y la Mujer, and Hospital San José, Queretaro, Mexico (a national referral centers for fetal surgery). Detailed fetal morphological ultrasound assessment, advanced neurosonography, maternal psychological evaluation, and fetal karyotype by amniocentesis were performed in all cases.

All ultrasound examinations were performed with Voluson E8 Expert BT 12.0 or Voluson E10 BT18.0 US equipment with a 6- to $2-\mathrm{MHz}$ linear curved-array transducer by 1 of 2 experienced examiners (R.C.M. or M.M.R.). The inclusion criteria for fetal surgery were singleton pregnancies at $<28$ weeks of gestation with $\mathrm{SB}$ with an upper anatomical level between $\mathrm{T} 1$ and $\mathrm{S} 1$ and evidence of hindbrain herniation (Arnold-Chiari II malformation). Fetal ventriculomegaly was defined as those fetuses with a mean lateral ventricle diameter above $10 \mathrm{~mm}$

The exclusion criteria were (a) additional structural malformations, (b) chromosomal abnormalities, (c) fetal kyphosis or scoliosis above $30^{\circ}$, (d) short cervical length ( $\left.<25 \mathrm{~mm}\right),($ e) 2 previous cesarean sections, (f) multiple uterine leiomyomas, (g) placenta previa, (h) acute or chronic maternal disease that may increase the surgical risk, (i) potential attachment difficulties at maternal psychological evaluation, and (j) positive maternal serology for HIV or hepatitis B or C. According to our clinical protocol, vaginal and urine cultures were performed 1 week before fetal surgery, and if positive, treatment was ensured.

The surgical protocol was approved by the hospital ethics committee (IRB 077/26042017). Following counseling regarding the prognosis of the fetal findings and the risks associated with the suggested fetal intervention, management options were discussed with the parents: expectant management during pregnancy with neonatal surgical repair or intrauterine SB repair through open fetal surgery. Termination of pregnancy was not considered an option since it is not legal in our country after the first trimester.

\section{Open Fetal Surgery}

Our first 2 surgeries were performed in collaboration with specialists from Santiago, Chile, led by Professor Waldo Sepulveda, who at that time had performed 35 procedures and whose experience was recently published, including 58 consecutive interventions [6]. The following interventions were performed by our local Mexican group, which included a multidisciplinary team involving maternal-fetal medicine specialists (M.M.R., J.L.G., and H.L.B.), fetal surgeon (R.C.M.), fetal sonographer (A.G.V.), pediatric neurosurgeon with previous experience in pediatric neuroendoscopical procedures (F.C.O.), and 2 of 3 anesthesiologists (K.A.V., J.C.V., and E.A.A.). The first cases were performed with the classic open fetal surgery technique previously described in the 

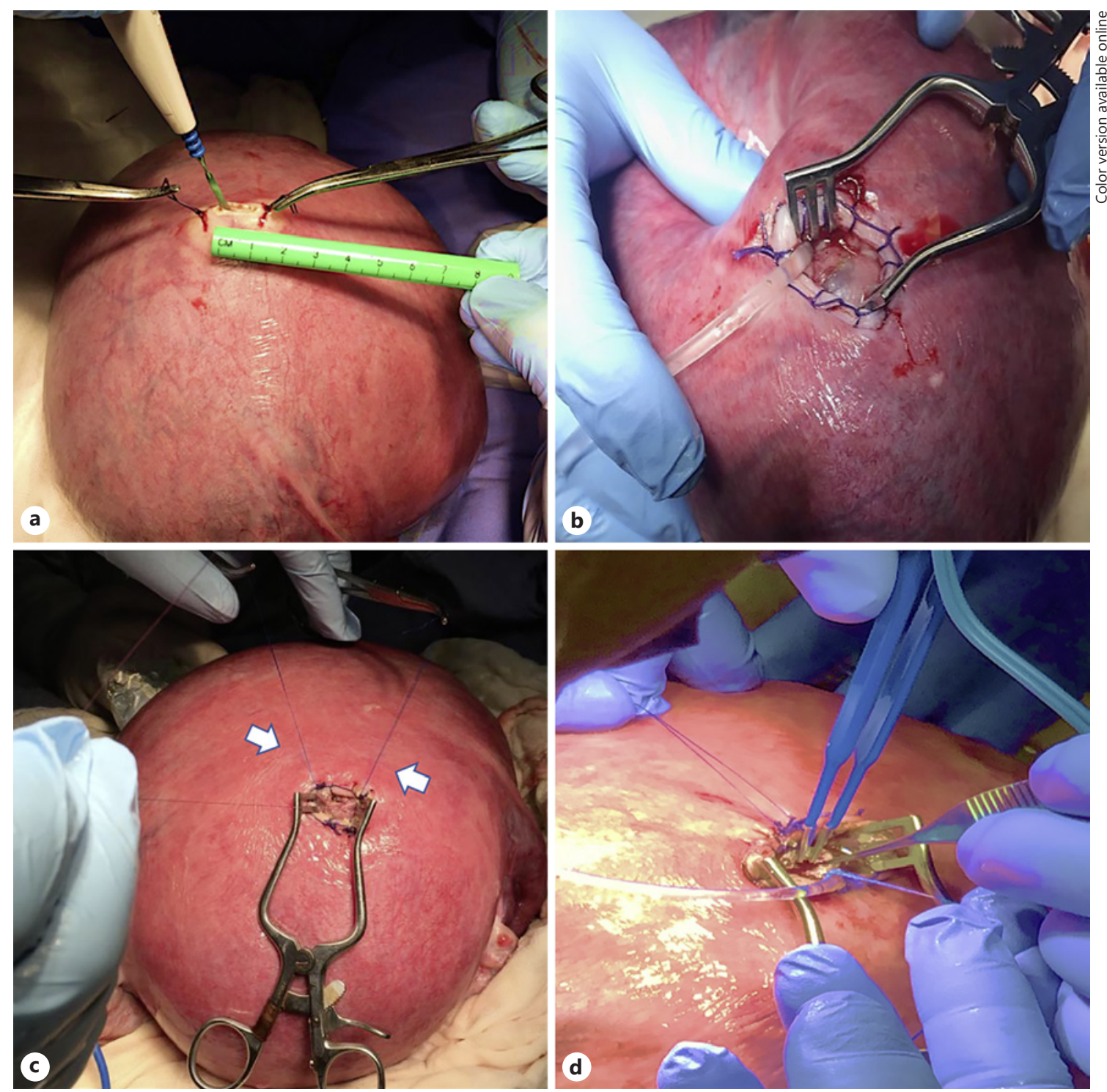

Fig. 1. 15-mm hysterotomy diameter (a) and placement of the self-retaining retractor allowing visualization of the neural defect and positioning of the enteral feeding tube for continuous infusion of warmed lactated Ringer's solution (b). Two lateral sutures (c) were placed in the fetal back (arrow) in order to be fixed against the hysterotomy and thus, to expose the portion of the neural tube defect required by the neurosurgeon to perform the fetal myeloplasty with a complete 3-layer correction (d).

MOMs trial (i.e., complete 3-layer correction through a 6- to 8-cm hysterotomy with leakage of amniotic fluid which was replaced before uterine closure) while in the last cases, a novel technique called open fetal microneurosurgery was performed.

In brief, under maternal regional and general anesthesia with sevoflurane, a maternal Pfannenstiel laparotomy through a low transverse abdominal incision was performed to allow uterine exteriorization from the abdominal cavity. The fetal position was evaluated by ultrasound and, if necessary, the fetus was moved by a gentle external manipulation to reach the optimal position, that is, a longitudinal position, with cephalic presentation and with the
SB defect located against the uterine wall and in the contralateral side of the placenta. Under ultrasound guidance, two 1-0 absorbable monofilament stitches were placed to plicate the membranes to the uterine wall and then a $15-$ to $20-\mathrm{mm}$ vertical and fundal midline hysterotomy was performed between the sutures with a monopolar cautery pencil (Fig. 1a). The amniotic membranes were opened and plicated to the uterine wall by continuous locking polyglactin 1-0 stitches (Ethicon Inc.). A self-retaining retractor (Weitlaner), which includes $3 \times 4$ blunt outward-curving teeth, was used to expose and spread the surgical site and, an $8 \mathrm{~F}$ enteral feeding tube was introduced to the amniotic cavity to perform a 

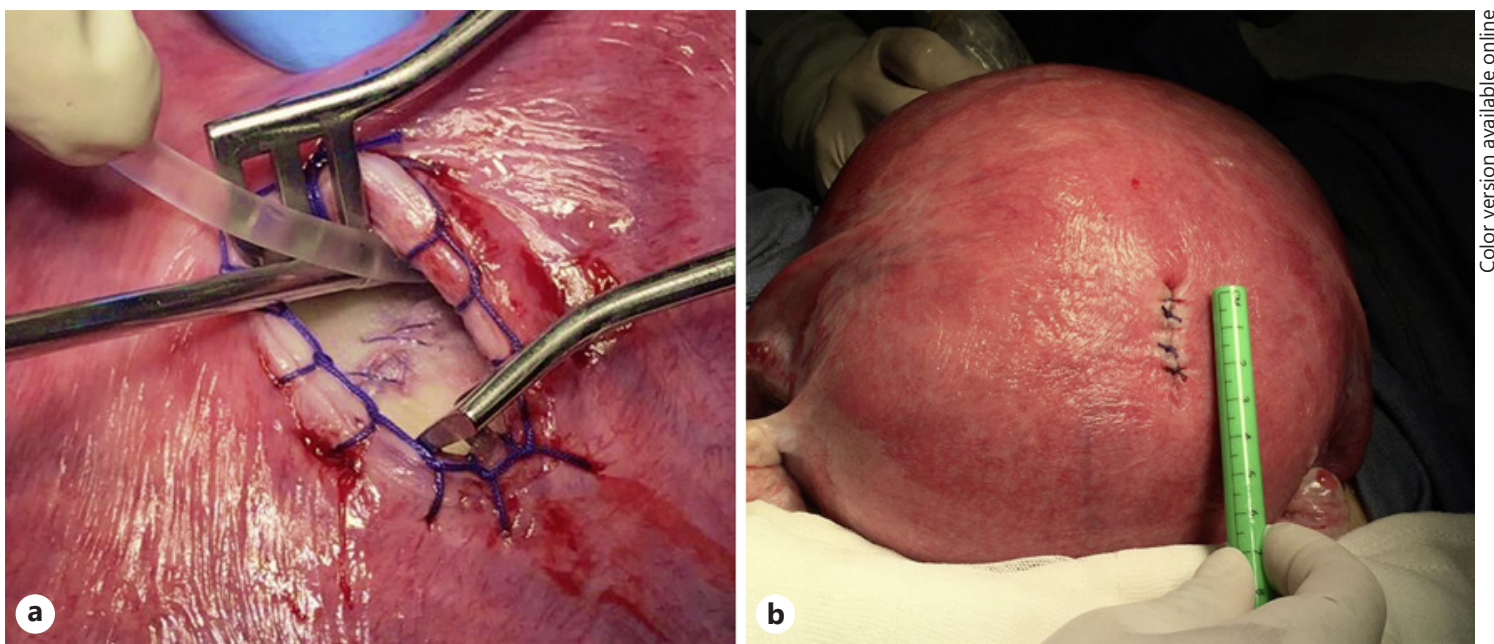

Fig. 2. Final result after multilayer fetal myeloplasty immediately before (a) and after (b) uterine closure.

continuous infusion of warmed $\left(37^{\circ} \mathrm{C}\right)$ lactated Ringer's solution with antibiotic ( 2 g cefotaxime per $\mathrm{L}$ ) in order to maintain the intrauterine volume and achieve a normal amniotic fluid index during the entire fetal surgery (Fig. 1b). To minimize fetal manipulation and amniotic fluid loss through the tiny uterine incision, the fetal back was fixed against the hysterotomy with 2 monofilament 4-0 stitches (Fig. 1c). Due to the small hysterotomy diameter, either an $\mathrm{HD}-3 \mathrm{D}$ exoscope system or surgical loupes $(4.5 \times)$ were used by the pediatric neurosurgeon (FCO) to improve illumination, magnification, and visualization of the neural placode, which was released and positioned within the spina canal and then a 3-layer watertight closure of the neural defect was performed using a monofilament 4-0 suture with a $17-\mathrm{mm}$ needle and biopolar cautery (Fig. 1d). In all cases, the size of the neural defect was larger than the hysterotomy incision, and therefore, exposure of the neural defect was mainly allowed by a gentle mobilization of the uterine retractor while the fetal back was held against the hysterotomy. In case this maneuver was insufficient, a gentle manipulation of the fetus by the fetal surgery specialist was performed to properly expose the portion of the neural tube defect required by the neurosurgeon. In cases with myeloschisis, a $2.5 \times 2.5-\mathrm{cm}$ biological patch (Lyoplant Onlay) was used, which is an absorbable, liquidtight, bilayer collagen implant produced from a lyophilized bovine pericardium designed for dura substitution that can be cut according to the shape of the neural defect. Fetal monitoring was continuously performed by a fetal sonographer during the entire surgical procedure by using fetal Doppler ultrasound. In case of uterine contraction during fetal intervention, maternal nitroglycerin was used for uterine relaxation [12]. Once fetal myeloplasty was completed, uterine closure was performed by 2 layers (Fig. 2), the uterus was interiorized into the abdominal cavity, and then the abdominal wall was closed in 3 layers.

\section{Maternal and Fetal Follow-Up}

After fetal surgery, the pregnant woman was hospitalized in the intensive care unit for $24 \mathrm{~h}$. An elastomeric pump was used for continuous infusion of pain drugs through the epidural catheter during the first $48 \mathrm{~h}$ after surgery. Prophylactic tocolysis with indomethacin, nifedipine or atosiban was administered immediately after wound closure and up to $72 \mathrm{~h}$ after the procedure. All patients were discharged 1 week after fetal surgery and were advised to stay in the city until delivery. The potential existence of uterine contractions was registered every $24 \mathrm{~h}$ by ambulatory cardiotocography. Whenever preterm delivery or PPROM was anticipated, a course of corticosteroids (betamethasone, $12 \mathrm{mg} 2$ doses $24 \mathrm{~h}$ apart) was administrated. Ultrasound examination to evaluate the amount of amniotic fluid, absence of chorioamniotic separation, fetal estimated weight, and cervical length was performed every week until delivery. If a short cervical length was observed during fetal follow-up, vaginal progesterone (200 mg daily) was administered until delivery. Fetal neurosonography to identify the regression of hindbrain herniation and to monitor the lateral ventricle diameters was also performed every week. Reversal of Chiari malformation was defined by fetal ultrasound when the obliterated cisterna magna became visible and measurable and the cerebellum fastigium regressed to a normal position and morphology, that is, above the foramen magnum.

\section{Neonatal Management and Infant Follow-Up}

According to our clinical protocol, cesarean delivery was planned at above 38 weeks. All deliveries and neonatal management were attended by staff obstetricians, maternal fetal medicine specialists, and neonatologists either at our center or at the referral center. Neurological follow-up was performed in our neurodevelopmental unit which includes neonatal neurohabilitation by a neonatal physical rehabilitator (A.C.B.) and clinical evaluations by a pediatric neurosurgeon, a pediatric orthopedist, and a pediatric urologist. Endoscopic third ventriculostomy was considered as primary treatment in all infants with hydrocephalus showing any in the following signs of intracranial hypertension: progressive increase in the head circumference accompanied with a bulging fontanelle (i.e., above the level of the bone as assessed when the baby is not crying and in an upright position), clinical signs of Chiari malformation (stridor, swallowing difficulties, apnea, and brady- 
Table 1. Maternal and fetal clinical characteristics of the selected pregnancies for intrauterine SB repair by classic open fetal surgery and open fetal microneurosurgery

\begin{tabular}{lccc}
\hline & $\begin{array}{l}\text { Classic open fetal } \\
\text { surgery, } \\
n=13\end{array}$ & $\begin{array}{l}\text { Open fetal } \\
\text { microneurosurgery, } \\
n=47\end{array}$ & $p$ value \\
\hline Maternal age, years & $31.4(7.7)$ & $29.5(6.1)$ & 0.36 \\
Primiparity, $n$ (\%) & $7(53.8)$ & $16(34.0)$ & 0.19 \\
Previous cesarean section, $n(\%)$ & $2(15.4)$ & $20(42.6)$ & 0.07 \\
BMI, kg/m ${ }^{2}$ & $25.2(4.2)$ & $24.9(3.9)$ & 0.81 \\
BMI $>$ 35, $n$ (\%) & $0(0)$ & $1(2.1)$ & 0.60 \\
Anterior placenta, $n(\%)$ & $10(76.9)$ & $17(36.2)$ & 0.01 \\
GA at diagnosis, wk & $22.5(2.2)$ & $22.0(2.5)$ & 0.53 \\
\hline MMC upper anatomical level, $n(\%)$ & & $0(0)$ & 0.06 \\
T12 & $1(7.7)$ & $5(10.6)$ & 0.75 \\
L1-L2 & $1(7.7)$ & $18(38.3)$ & 0.99 \\
L3-L4 & $5(38.5)$ & $14(29.8)$ & 0.55 \\
L5 & $5(38.5)$ & $10(21.3)$ & 0.26 \\
S1 & $1(7.7)$ & $8(17.0)$ & 0.42 \\
Myeloschisis & $1(7.7)$ & $33(70.2)$ & 0.64 \\
Ventriculomegaly & $10(76.9)$ & $12.0(3.2)$ & 0.45 \\
Lateral ventricle diameter, mm & $24(51.1)$ & 0.24 \\
Club foot or feet, $n$ (\%) & $12.6(2.3)$ & $9(69.2)$ & \\
\hline
\end{tabular}

Results are expressed as mean (standard deviation) and percentages. GA, gestational age; MMC, myelomeningocele; SB, spina bifida.

cardia), or persistent cerebrospinal fluid leakage from the MMC wound or bulging at the repair site. Ventriculoperitoneal shunt placement was considered when there was an anatomical impossibility to perform third ventriculostomy during neuroendoscopy [13].

\section{Statistical Analysis}

Data were collected in an Access file for Windows 2010 (Microsoft Corp., Redmond, WA, USA). Student's $t$ test and the Pearson $\chi^{2}$ test were used to compare quantitative and qualitative data within the study group, respectively. All tests were 2-tailed, and a probability value of $<0.05$ was considered statistically significant. Statistical calculations and descriptive analysis were performed using Statistical Package for the Social Sciences (SPSS 25.0, SPSS Inc., Chicago, IL, USA) software.

\section{Results}

A total of 220 fetuses with SB were evaluated during the 3.5-year study period. And, 163 patients did not fulfill the criteria for fetal intervention. The main exclusion criterion was diagnosis and/or referral above 28 weeks of gestation (35.5\%). Thus, 61 cases $(27.7 \%)$ were selected for intrauterine SB repair. In the first 13 cases, intrauterine $\mathrm{SB}$ repair was successfully performed using the classic open fetal surgery technique. Among the last 48 selected cases, 1 case was abandoned before performing maternal laparotomy due to anaphylactic shock associated with maternal cardiac arrest and fetal bradycardia, which was promptly resolved with cardiopulmonary resuscitation. She had an uneventful cesarean delivery at 39 weeks. In the remaining 47 consecutive cases, fetal myeloplasty with a complete 3-layer correction was successfully performed by open fetal microneurosurgery. There were not any cases requiring conversion from microneurosurgery to the classic larger uterine incision and therefore, a total of 47 cases treated with open microneurosurgery were compared with 13 controls repaired using the classic open fetal surgery. Baseline maternal and fetal characteristics of the 2 study groups are shown in Table 1. Table 2 shows the perinatal outcomes of the 55 women who have delivered by the time of analysis. No significant differences were observed in the upper level of the spinal defect, gestational age (GA) at fetal intervention ( 25.3 vs. 25.1 weeks, $p=0.38$ ), surgical times (107 vs. $120 \mathrm{~min}, p=0.15$ ), and rate of myeloschisis ( 17.0 vs. $7.0 \%, p=0.42$ ) between both groups. After fetal intervention, the group with open microneurosurgery showed a significantly lower rate of oligohydramnios ( 0 vs. $15.4 \%, p=0.01$ ), and PPROM 
Table 2. Maternal and fetal studied variables from fetal surgery to delivery

\begin{tabular}{|c|c|c|c|}
\hline & $\begin{array}{l}\text { Classic open } \\
\text { fetal surgery, } \\
n=13\end{array}$ & $\begin{array}{l}\text { Open fetal } \\
\text { microneurosurgery, } \\
n=42\end{array}$ & $p$ value \\
\hline GA at fetal surgery, wk & $25.1(1.0)$ & $25.3(0.82)$ & 0.38 \\
\hline Surgery time, min & $120.6(25.4)$ & $107.3(30.3)$ & 0.15 \\
\hline Maternal hospital days & $6.2(1.7)$ & $5.6(1.2)$ & 0.32 \\
\hline Postsurgical maternal pulmonary edema, $n(\%)$ & $0(0)$ & $1(2.4)$ & 0.57 \\
\hline Maternal bleeding requiring blood transfusion, $n(\%)$ & $1(7.7)$ & $0(0)$ & 0.07 \\
\hline Chorioamnionitis, $n(\%)$ & $1(7.7)$ & $2(4.8)$ & 0.68 \\
\hline Placental abruption, $n(\%)$ & $1(7.7)$ & $0(0)$ & 0.07 \\
\hline Oligohydramnios, $n(\%)$ & $2(15.4)$ & $0(0)$ & 0.01 \\
\hline Chorioamniotic separation, $n(\%)$ & $4(30.8)$ & $9(21.4)$ & 0.49 \\
\hline PPROM, $n(\%)$ & $7(53.8)$ & $8(19.0)$ & 0.01 \\
\hline PPROM GA, wk & $30.0(4.2)$ & $31.2(2.9)$ & 0.41 \\
\hline Intrauterine fetal demise, $n(\%)$ & $1(7.7)$ & $0(0)$ & 0.07 \\
\hline GA at birth, wk & $32.7(4.1)$ & $35.4(3.0)$ & 0.03 \\
\hline Interval between fetal surgery and delivery, weeks & $7.5(3.8)$ & $9.7(3.0)$ & 0.04 \\
\hline Cesarean delivery, $n(\%)$ & $13(100)$ & $39(92.9)$ & 0.32 \\
\hline Intact hysterotomy site, $n(\%)$ & $13(100)$ & $42(100)$ & 1.00 \\
\hline Preterm delivery <37 wk, $n(\%)$ & $10(76.9)$ & $22(52.4)$ & 0.12 \\
\hline Preterm delivery <34 wk, $n(\%)$ & $8(61.5)$ & $9(21.4)$ & 0.01 \\
\hline Preterm delivery <32 wk, $n(\%)$ & $4(30.8)$ & $5(11.9$ & 0.11 \\
\hline Preterm delivery <30 wk, $n(\%)$ & $3(23.1)$ & $3(7.1)$ & 0.11 \\
\hline Birth weight, $g$ & $1,992(724)$ & $2,207(656)$ & 0.32 \\
\hline Neonatal death, $n(\%)$ & $2(15.4)$ & $2(4.8)$ & 0.20 \\
\hline Perinatal death, $n(\%)$ & $3(23.1)$ & $2(4.8)$ & 0.04 \\
\hline Reversal of Chiari II malformation, $n(\%)$ & $6(46.2)$ & $26(61.9)$ & 0.31 \\
\hline Increase fetal cerebral lateral ventricle size, $n(\%)$ & $7(53.8)$ & $21(50.0)$ & 0.81 \\
\hline
\end{tabular}

Results are expressed as mean (standard deviation) or percentages. GA, gestational age; PPROM, preterm premature rupture of membranes.

( 19.0 vs. $53.8 \%, p=0.01$ ), higher GA at birth (35.4 vs. 32.7, weeks, $p<0.05$ ), higher interval between fetal intervention and delivery ( 9.7 vs. 7.5 weeks, $p<0.05$ ), lower rate of preterm delivery below 34 weeks ( 21.4 vs. $61.5 \%, p=$ $0.01)$, and lower rate of perinatal death ( 4.8 vs. $23.1 \%, p=$ 0.04 ) than the group with classic open surgery. No differences were observed in the rate of maternal complications such as placental abruption, pulmonary edema, chorioamnionitis, or maternal bleeding requiring blood transfusion.

Within the group with open microneurosurgery, 3 patients $(7.1 \%)$ experienced intense uterine contractions that required an emergency but uneventfully vaginal delivery. All patients showed intact hysterotomy site at delivery, and there were no maternal deaths in any group. Table 3 illustrates the infant characteristics during the follow-up among survivors. The rate of hydrocephalus requiring either ventriculoperitoneal shunting (7.5 vs.
$20 \%, p=0.24)$ or only endoscopic third ventriculostomy ( 12.5 vs. $20 \%, p=0.54$ ) was similar between both groups. There were no neonates with skin dehiscence or skin lesions attributable to the sutures placed to fix fetal back. The rate of subcutaneous cerebrospinal fluid leakage requiring a complementary postnatal surgical repair was similar between both groups ( 5.0 vs. $20.0 \%, p=0.12$ ). Details from patients fulfilling MOMs criteria for fetal surgery are provided in Table 4, which shows similar results compared with those observed in the whole population.

\section{Discussion}

This is the first study highlighting that intrauterine open neural tube defect repair is feasible by open fetal microneurosurgery. This is a novel fetal surgery approach for SB repair, which is based on a reduced 15-20-mm hys- 
Table 3. Infant characteristic during follow-up among survivors

\begin{tabular}{lccc}
\hline & $\begin{array}{l}\text { Classic open } \\
\text { fetal surgery, } \\
n=10\end{array}$ & $\begin{array}{l}\text { Open fetal } \\
\text { microneurosurgery, } \\
n=40\end{array}$ & $p$ value \\
\hline Infant age at last evaluation, months & $32.5(5.1)$ & $12.2(9.1)$ & $<0.01$ \\
Infant age $>1$ year, $n$ (\%) & $10(100)$ & $18(45.0)$ & $<0.01$ \\
Hydrocephaly, $n$ (\%) & $4(40)$ & $8(20.0)$ & 0.19 \\
Ventriculoperitoneal shunting, $n(\%)$ & $2(20.0)$ & $37.0(32.8)$ & 0.24 \\
Infant age at shunting, days & $20.5(16.3)$ & $5(12.5)$ & 0.57 \\
Ventriculostomy by neuroendoscopy, $n(\%)$ & $2(20.0)$ & $11.0(3.2)$ & 0.54 \\
Infant age at neuroendoscopy, months & $31.6(34.5)$ & $0(0)$ & 1.00 \\
Neonatal skin dehiscence, $n(\%)$ & $0(0)$ & $2(5.0)$ & 0.12 \\
Cerebrospinal fluid leakage after birth, $n(\%)$ & $2(20.0)$ & $8(20.0)$ & 0.12 \\
Postnatal surgery, $n$ (\%) & $2(20.0)$ & $1(10)$ & 0.46 \\
Absent inferior limb movements, $n(\%)$ & & $2(5.0)$ & 0.25 \\
\hline
\end{tabular}

Results are expressed as mean (standard deviation) and percentage.

Table 4. Maternal and fetal studied variables from patients fulfilling GA within MOMs criteria for intrauterine SB repair

\begin{tabular}{|c|c|c|c|}
\hline & $N=13$ & $N=30$ & $p$ value \\
\hline GA at fetal surgery, wk & $25.1(1.0)$ & $24.9(0.54)$ & 0.49 \\
\hline Surgery time, $\min$ & $120(25.4)$ & $108(31.3)$ & 0.24 \\
\hline Maternal hospital days & $6.2(1.7)$ & $5.6(1.1)$ & 0.18 \\
\hline Postsurgical maternal pulmonary edema, $n(\%)$ & $0(0)$ & $0(0)$ & 1.00 \\
\hline Maternal bleeding requiring blood transfusion, $n(\%)$ & $1(7.7)$ & $0(0)$ & 0.12 \\
\hline Chorioamnionitis, $n(\%)$ & $1(7.7)$ & $2(6.7)$ & 0.90 \\
\hline Placental abruption, $n(\%)$ & $1(7.7)$ & $0(0)$ & 0.12 \\
\hline Oligohydramnios, $n(\%)$ & $2(15.4)$ & $0(0)$ & 0.03 \\
\hline Chorioamniotic separation, $n(\%)$ & $4(30.8)$ & $5(16.7)$ & 0.29 \\
\hline PPROM, $n(\%)$ & $7(53.8)$ & $5(16.7)$ & 0.01 \\
\hline PPROM GA, wk & $30.0(4.2)$ & $30.6(3.3)$ & 0.75 \\
\hline Interval between fetal surgery and delivery, weeks & $7.5(3.8)$ & $10.0(3.3)$ & 0.03 \\
\hline GA at birth, wk & $32.7(4.1)$ & $35.0(3.3)$ & 0.05 \\
\hline Cesarean delivery, $n(\%)$ & $13(100)$ & $28(93.3)$ & 0.34 \\
\hline Intact hysterotomy site, $n(\%)$ & $13(100)$ & $30(100)$ & 1.00 \\
\hline Preterm delivery <37 wk, $n(\%)$ & $10(76.9)$ & $14(46.7)$ & 0.06 \\
\hline Preterm delivery <34 wk, $n(\%)$ & $8(61.5)$ & $7(23.3)$ & 0.01 \\
\hline Preterm delivery <32 wk, $n(\%)$ & $4(30.8)$ & $5(16.7)$ & 0.29 \\
\hline Birth weight, g & $1,992(724)$ & $2,127(633)$ & 0.54 \\
\hline Perinatal death, $n(\%)$ & $3(23.1)$ & $2(6.7)$ & 0.12 \\
\hline Reversal of Chiari II malformation, $n(\%)$ & $6(46.2)$ & $20(66.7)$ & 0.21 \\
\hline Increase fetal cerebral lateral ventricle size, $n(\%)$ & $7(53.8)$ & $14(46.7)$ & 0.67 \\
\hline Infant age at last evaluation, months & $32.5(5.1)$ & $12.7(9.6)$ & $<0.01$ \\
\hline Infant age $>1$ year & $10 / 10(100 \%)$ & $13 / 28(46.4 \%)$ & $<0.01$ \\
\hline Hydrocephaly & $4 / 10(40 \%)$ & $5 / 28(17.9 \%)$ & 0.16 \\
\hline Ventriculoperitoneal shunting & $2 / 10(20 \%)$ & $2 / 28(7.1 \%)$ & 0.25 \\
\hline Ventriculostomy by neuroendoscopy & $2 / 10(20 \%)$ & $3 / 28(10.7 \%)$ & 0.46 \\
\hline Neonatal skin dehiscence, $n(\%)$ & $0(0)$ & $0(0)$ & 1.00 \\
\hline Cerebrospinal fluid leakage at birth & $2 / 10(20 \%)$ & $2 / 28(7.1 \%)$ & 0.25 \\
\hline Postnatal surgery & $2 / 10(20 \%)$ & $2 / 28(7.1 \%)$ & 0.25 \\
\hline Absent inferior limb movements & $1 / 10(10 \%)$ & $6 / 28(21.4 \%)$ & 0.42 \\
\hline
\end{tabular}

GA, gestational age; PPROM, preterm premature rupture of membranes; SB, spina bifida. 
terotomy, decreased fetal manipulation, and maintenance of a normal amniotic fluid and uterine volume during the whole fetal intervention and was associated with better perinatal outcomes, lower rate of preterm delivery, PPROM, and perinatal death compared to the classic open fetal surgery technique.

Classic open fetal surgery was initially performed through a 6- to 8-cm hysterotomy, allowing the pediatric neurosurgeon a good visualization for a multilayer correction of the neural defect [3]. However, such technique has showed potential risks of maternal morbidity and complications such as PPROM, preterm delivery, placental abruption, chorioamniotic separation, chorioamnionitis, perinatal death, maternal pulmonary edema, uterine bleeding requiring maternal blood transfusion, and uterine dehiscence at delivery $[4,5]$. The concept of decreasing the hysterotomy length for MMC repair in an attempt to decrease maternal morbidity and perinatal complications was first reported by Botelho et al. [10]. In such study, the outcomes of 39 consecutive fetuses managed with open fetal surgery through a $2.5-3.5 \mathrm{~cm}$ hysterotomy were evaluated showing a $50 \%$ decreased risk of PPROM and preterm delivery, with no maternal, fetal or neonatal complications. In agreement with such study, the PPROM rate, preterm delivery rate, and short-term neonatal outcomes in our microneurosurgery technique compare favorably against those in our control group and with the results reported in previous published studies (Table 5).

In a small cohort of MMC cases, the team at Texas Children's Hospital recently reported a novel fetoscopic technique using a two-port approach with an exteriorized uterus showing good perinatal outcomes with lower rate of preterm delivery and PPROM than previous published techniques [14-17]. In terms of uterine invasion, since our technique requires a hysterotomy of only $15-20 \mathrm{~mm}$, it could be argued that our technique may be as minimally invasive as the fetoscopic approach which requires 2 or 3 ports of $5 \mathrm{~mm}$, that is, 2 or 3 uterine incisions of at least $5 \mathrm{~mm}$ each. However, our technique had required lower surgical time than all fetoscopic techniques (Table 5) [8, $9,14-16]$. Another potential strength of our technique over the fetoscopic approach is that it does not require the use of carbon dioxide insufflation into the amniotic cavity, and thus avoidance its potential theoretical risks [18, 19]. In addition, our results also suggest that decreasing the hysterotomy diameter, minimizing fetal manipulation, and maintaining normal amniotic fluid volume during fetal surgery may be associated with a lower incidence of oligohydramnios during fetal follow-up. While no cases of fetal oligohydramnios were observed neither in our microneurosurgery technique nor in Botelho's study with minihysterotomy, the classic open fetal surgery and all fetoscopic techniques have showed oligohydramnios rates of 23 and 14\%, respectively [7, 14-17, 20].

Although we expect that minimizing the uterine incision may also improve reproductive outcomes by reducing the risk of uterine rupture and the rate of cesarean section, other obstetrical parameters such as parity, bishop score, labor stage, fetal estimated weight, and fetal position must be also considered for an algorithm for a clinical decision regarding vaginal birth in this population. Notably, a few patients treated with our technique showed an unplanned but uneventfully vaginal delivery. This observation may represent the basis for further studies evaluating the possibility of a planned vaginal delivery in selected cases with open microsurgery.

Our study supports the current evidence demonstrating that fetal surgery for SB repair decreases the risk of hydrocephalus and the need of ventriculoperitoneal shunting, with a low risk of perinatal death $[3,11,20,21]$. We recognize that our study has also some limitations. First of all, although similar or higher than that in previous published studies, our included sample size is limited, and further studies are required for an external validation of our results. The lack of long-term neurological outcomes is also a limitation, and therefore, a long-term study is required to confirm the potential benefits and neurological outcomes of our minimally invasive neurosurgical intervention. Of note and contrary to the MOMs trial, cases with diagnosis or referral between 26 and 28 weeks of gestation were also selected for fetal intervention. Although, this is not the first published study including cases above 26 weeks of gestation $[10,11,15,16$, 22], a previous study by Peralta et al. [11] showed that early GA at surgery was associated with higher rates of hindbrain herniation reversal and lower frequency of ventriculoperitoneal shunting, and therefore, it would be expected that fetal interventions above 26 weeks may be associated with poorer neurological outcomes, but further comparison studies between early and late surgical procedures are required to justify the upper limit of GA at surgery. In addition, it could be argued that a potential risk of trauma to the placode may exist during fetal manipulation used to expose the neural tube defect through a tiny uterine incision. However, the fact that amniotic membranes were plicated against myometrium makes such risk very unlikely. Finally, as a potential limitation for the widespread use of this novel approach for intrauterine SB repair, it must be acknowledged that such minimally invasion procedure is very challenging be- 


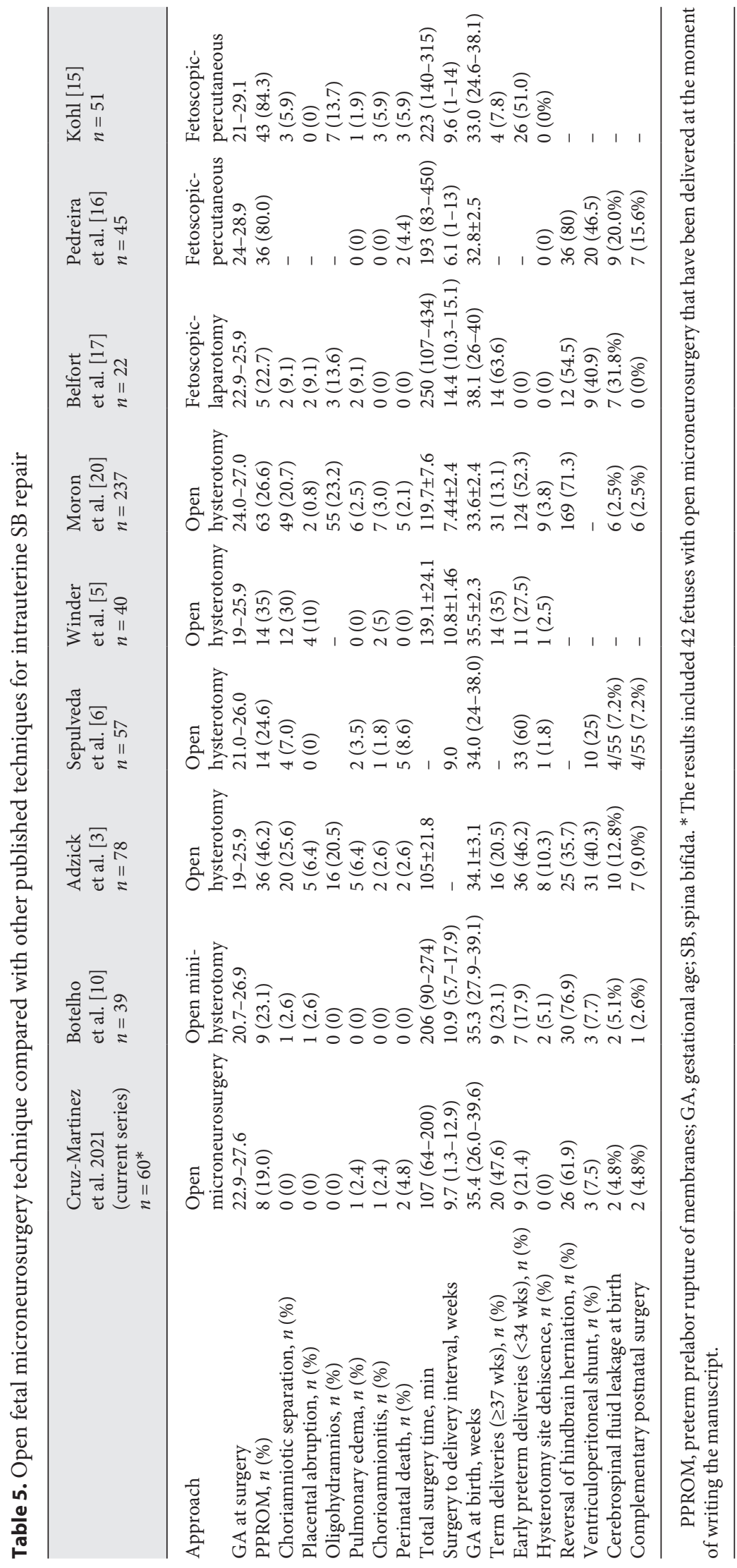


cause it reduces visualization of the surgical field and thus requires advanced training of the neurosurgeon. Nevertheless, using different surgical tools such as the self-retaining retractor allows for spreading the surgical site to about $5 \mathrm{~mm}$ and increases maneuvering through the smaller uterine access diameter. In addition, using the exoscopic system or surgical loupes enhanced pediatric neurosurgeon enhanced surgical field understanding and improved hand-eye coordination for a proper prenatal surgical correction of SB [23], but we learned that using surgical loupes, which are cheaper than the exoscopic system, was enough to improve magnification and visualization of the surgical field on this high-precision neurosurgical procedure. In conclusion, our initial experience suggests that open fetal microneurosurgery for neural tube defect repair is feasible and is associated with better perinatal outcomes and similar short-term neurological outcomes than the classic open fetal surgery technique.

\section{Acknowledgements}

Rogelio Cruz-Martinez was supported by the National Council of Science and technology (Conacyt) and wishes to thank Prof. Waldo Sepulveda, Felipe Otayza, and Juan Carlos Devoto (FETALMED- Fetal Surgery group, Santiago, Chile) for supporting the beginning of the national Mexican program of open fetal surgery for intrauterine SB repair.

\section{Statement of Ethics}

Our research complies with the guidelines for human studies and was conducted ethically in accordance with the World Medical Association Declaration of Helsinki. All subjects gave their written informed consent, and the study protocol was approved by the by the Hospital Ethics Committee (Hospital de Especialidades del Niño y la Mujer Dr. Felipe Núñez Lara, Querétaro, México IRB 077/26042017).

\section{Conflict of Interest Statement}

The authors have no conflicts of interests to declare.

\section{Funding Sources}

No funding was obtained for the completion of this study.

\section{Author Contributions}

R.C.M., F.C.O., M.M.R., K.A.V., A.G.V., J.L.G., H.L.B., J.C.V., and E.A.A. participated in all the fetal interventions. R.C.M., F.C.O., M.M.R., and K.A.V. made substantial contributions to design the novel fetal surgery technique. R.C.M., F.C.O., M.M.R., A.C.V., E.C.G., and I.J.M. did the acquisition of data. R.C.M., F.C.O., M.M.R., and E.C.G. did the analysis and interpretation of data. R.C.M. and R.V.G. drafted the manuscript. All authors listed revised the article for important intellectual content and gave final approval of the final version for publication.

\section{References}

1 Mitchell LE, Adzick NS, Melchionne J, Pasquariello PS, Sutton LN, Whitehead AS. Spina bifida. Lancet. 2004 Nov 20-26; 364(9448):1885-95.

2 Bruner JP, Tulipan N, Paschall RL, Boehm FH, Walsh WF, Silva SR, et al. Fetal surgery for myelomeningocele and the incidence of shunt-dependent hydrocephalus. JAMA. 1999 Nov 17;282(19):1819-25.

3 Adzick NS, Thom EA, Spong CY, Brock JW 3rd, Burrows PK, Johnson MP, et al. A randomized trial of prenatal versus postnatal repair of myelomeningocele. N Engl J Med. 2011 Mar 17;364(11):993-1004.

4 Sacco A, Van der Veeken L, Bagshaw E, Ferguson C, Van Mieghem T, David AL, et al. Maternal complications following open and fetoscopic fetal surgery: a systematic review and meta-analysis. Prenat Diagn. 2019 Mar; 39(4):251-68.

5 Winder FM, Vonzun L, Meuli M, Moehrlen U, Mazzone L, Krähenmann F, et al. Maternal complications following open fetal myelomeningocele repair at the zurich center for fetal diagnosis and therapy. Fetal Diagn Ther. 2019;46(3):153-8.

6 Sepulveda W, Corral E, Alcalde JL, Otayza F, Müller JM, Ravera F, et al. Prenatal repair of spina bifida: a 2-center experience with open intrauterine neurosurgery in Chile. Fetal Diagn Ther. 2020;47(12):873-81.

7 Sacco A, Ushakov F, Thompson D, Peebles D, Pandya P, De Coppi P, et al. Fetal surgery for open spina bifida. Obstet Gynaecol. 2019 Oct; 21(4):271-82.

8 Araujo Junior E, Eggink AJ, van den Dobbelsteen J, Martins WP, Oepkes D. Procedurerelated complications of open vs endoscopic fetal surgery for treatment of spina bifida in an era of intrauterine myelomeningocele repair: systematic review and meta-analysis. Ultrasound Obstet Gynecol. 2016 Aug;48(2): $151-60$.

9 Joyeux L, Engels AC, Russo FM, Jimenez J, Van Mieghem T, De Coppi P, et al. Fetoscopic versus open repair for spina bifida aperta: a systematic review of outcomes. Fetal Diagn Ther. 2016;39(3):161-71.
10 Botelho RD, Imada V, Rodrigues da Costa KJ Watanabe LC, Rossi Júnior R, De Salles AAF, et al. Fetal myelomeningocele repair through a mini-hysterotomy. Fetal Diagn Ther. 2017; 42(1):28-34.

11 Peralta CFA, Botelho RD, Romano ER, Imada V, Lamis F, Junior RR, et al. Fetal open spinal dysraphism repair through a minihysterotomy: influence of gestational age at surgery on the perinatal outcomes and postnatal shunt rates. Prenat Diagn. 2020;40(6): 689-97.

12 Devoto JC, Alcalde JL, Otayza F, Sepulveda W. Anesthesia for myelomeningocele surgery in fetus. Childs Nerv Syst. 2017 Jul;33(7): 1169-75.

13 Elbabaa SK, Gildehaus AM, Pierson MJ, Albers JA, Vlastos EJ. First 60 fetal in-utero myelomeningocele repairs at Saint Louis Fetal Care Institute in the post-MOMS trial era: hydrocephalus treatment outcomes (endoscopic third ventriculostomy versus ventriculoperitoneal shunt). Childs Nerv Syst. 2017 Jul; 33(7):1157-68. 
14 Degenhardt J, Schürg R, Winarno A, Oehmke F, Khaleeva A, Kawecki A, et al. Percutaneous minimal-access fetoscopic surgery for spina bifida aperta. Part II: maternal management and outcome. Ultrasound Obstet Gynecol. 2014 Nov;44(5):525-31.

15 Kohl T. Percutaneous minimally invasive fetoscopic surgery for spina bifida aperta. Part I: surgical technique and perioperative outcome. Ultrasound Obstet Gynecol. 2014 Nov; 44(5):515-24.

16 Pedreira DA, Zanon N, Nishikuni K, Moreira de Sá RA, Acacio GL, Chmait RH, et al. Endoscopic surgery for the antenatal treatment of myelomeningocele: the CECAM trial. Am J Obstet Gynecol. 2016 Jan;214(1):111-e11.
17 Belfort MA, Whitehead WE, Shamshirsaz AA, Bateni $\mathrm{ZH}$, Olutoye OO, Olutoye OA, et al. Fetoscopic open neural tube defect repair: development and refinement of a two-port, carbon dioxide insufflation technique. Obstet Gynecol. 2017 Apr;129(4):734-43.

18 Skinner S, Crossley K, Amberg B, Kashyap A, Hooper S, Deprest JA, et al. The effects of partial amniotic carbon dioxide insufflation in an ovine model. Prenat Diagn. 2018 Dec;38(13): 994-1003.

19 Skinner S, DeKoninck P, Crossley K, Amberg B, Deprest J, Hooper S, et al. Partial amniotic carbon dioxide insufflation for fetal surgery. Prenat Diagn. 2018 Dec;38(13):983-93.

20 Moron AF, Barbosa MM, Milani H, Sarmento SG, Santana E, Suriano IC, et al. Perinatal outcomes after open fetal surgery for myelomeningocele repair: a retrospective cohort study. BJOG. 2018 Sep;125(10):1280-6.
21 Houtrow AJ, Thom EA, Fletcher JM, Burrows PK, Adzick NS, Thomas NH, et al. Prenatal repair of myelomeningocele and school-age functional outcomes. Pediatrics. $2020 \mathrm{Feb}$; 145(2):e20191544.

22 Corral E, Sepulveda W, Ravera F, Muller JM, Tapia M, Reascos $M$, et al. Use of plastic wound retractor at hysterotomy site in prenatal repair of myelomeningocele: a new technique. J Matern Fetal Neonatal Med. 2020; 33(17):3010-15.

23 Oertel JM, Burkhardt BW. Vitom-3D for exoscopic neurosurgery: initial experience in cranial and spinal procedures. World Neurosurg. 2017 Sep;105:153-62. 